\section{Was haben Kapitalanlagen und öffentliche Verkehrsmittel gemein?}

\author{
Erstmals wurde der Carbon Footprint für Kapitalanlage- \\ produkte in Deutschland berechnet. Die Ergebnisse zeigen, \\ dass die private Kapitalanlage in erheblichem Maße mit \\ Treibhausgasemissionen verknüpft ist. \\ Von Daniel Wendler, Walter Kahlenborn und Romy Fiedler
}

$\mathbf{V}_{\mathrm{p}}^{\mathrm{o}}$ om Kauf saisonaler Landwirtschaftsprodukte über die energieeffiziente Sanierung von Gebäuden bis hin zur Nutzung öffentlicher Verkehrsmittel: Für den Markt von klimafreundlichen Konsumgütern stellt der Carbon Footprint eine einfache Orientierungshilfe dar, um den Einfluss der konsumierten Produkte auf das Klima zu beurteilen. Kapitalanlageprodukte wurden bisher noch nicht abgebildet. Zum einen fehlt es derzeit an etablierten Verfahren, um den Carbon Footprint von Kapitalanlagen zu berechnen. Zum anderen ist eine öffentliche Auseinandersetzung mit dem Thema Klimaschutz und Finanzen erst in Ansätzen zu beobachten.

\section{Klimafreundliche Kapitalanlagen?}

Doch gerade der Finanzmarkt bietet einen wirkungsvollen Hebel für den Klimaschutz. Pro 10.000 investierten Euro werden derzeit fünf Tonnen Treibhausgasemissionen im Jahr mitfinanziert. Dies entspricht knapp der Hälfte des durchschnittlichen KohlenstoffdioxidFußabdruckes eines deutschen Bundesbürgers. Angesichts der erheblichen Kapitalbeträge, die deutsche Haushalte bereits angespart haben, wird die Frage, wie klimafreundlich dieses Geld investiert wird, künftig von großer Bedeutung sein. Denn Investitionsentscheidungen in wirtschaftliche Aktivitäten bestimmen in entscheidendem Maße die Treibhausgasemissionen der nächsten Jahre.
Wie unterscheiden sich klimafreundliche von konventionellen Kapitalanlagen? Und wie kann die Emissionsintensität der unterschiedlichen Kapitalanlageprodukte dargestellt werden? Um diese Fragen zu beantworten, hat adelphi zusammen mit Inrate erstmals das Instrument des Carbon Footprint auf gängige Kapitalanlageprodukte in Deutschland angewandt. Die Studie bietet damit eine erste Diskussionsgrundlage, wie über Kapitalanlageprodukte finanzierte Treibhausgasemissionen erfasst und analysiert werden können.

\section{Die Zusammensetzung des Carbon Footprint}

Anders als beim Kauf oder der Nutzung von Konsumprodukten werden für den Fußabdruck von Kapitalanlageprodukten die Emissionen summiert, die bei Unternehmungen und Projekten entstehen, an denen sich die Anleger mit ihrem Kapital finanziell beteiligen (1). Dabei wurde mit der angewandten Methodik darauf geachtet, dass nur der Teil der Emissionen eines Unternehmens in die Berechnung einfließt, den Anleger auch finanziell am Fremd- oder Eigenkapital bei der Unternehmensfinanzierung vertreten.

Die Analyse der 20 volumenstärksten Nachhaltigkeits- und Klimafonds in Deutschland zeigt dabei, dass diese Produkte die treibhausgasintensiven Werte stärker meiden als ihre konventionellen Pendants. Durchschnittlich sind Einspa- rungen um 46 Prozent bei den Nachhaltigkeitsfonds (605 THGg/Euro) und 30 Prozent bei den Klimafonds (776 THGg/Euro) gegenüber dem Durchschnitt der konventionellen Fonds (1.111 THGg/Euro) möglich.

Ein noch deutlicheres Bild ergibt sich bei der Analyse der Treibhausgasintensität von Unternehmensbeteiligungen in Form von Aktien. Ihr Carbon Footprint wurde über die Untersuchung gängiger Aktienindizes dargestellt. Demnach ist der Erwerb von Aktien nachhaltiger Unternehmen (631 THGg/Euro) mit 49 Prozent weniger Treibhausgasemissionen verknüpft, als Unternehmensaktien aus besonders marktrelevanten europäischen Standardindizes, die eine Treibhausgasintensität von 1243 THGg/Euro aufweisen.

Für durchschnittliche Sparprodukte ergibt sich nach den Berechnungen ein Carbon Footprint von 199 THGg/Euro. Dies ist deutlich weniger als bei den Aktienwerten. Denn bei Sparprodukten werden die Mittel der Anleger nicht nur zur Finanzierung von börsennotierten Aktienunternehmen eingesetzt, sondern dienen auch dem Hausbau, dem Kauf von Konsumgütern oder der Finanzierung von Staatsausgaben. Analog wurde auch die Treibhausgasintensität eines klimafreundlichen Sparanlageprodukts berechnet, welches Kunden die ausschließliche Verwendung ihrer Einlagen für die Finanzierung von Projekten mit erneuerbaren Energien garantiert. Hier ergibt sich eine deutlich geringere Emissionsintensität von 66 THGg pro Euro für das klimafreundliche Sparprodukt.

\section{Starke Variationen der Treibhausgasintensitäten}

Innerhalb der einzelnen Produktklassen ergibt sich eine starke Streuung der finanzierten Emissionswerte. Am Beispiel der untersuchten Aktienfonds zeigt sich, dass auch einzelne konventionelle Produkte derzeit mit deutlich geringeren Treibhausgasemissionen verknüpft sein können als bestimmte Nachhaltigkeitsoder Klimafonds. Auch ohne die Formulierung einer bewusst klimafreundlichen Anlagestrategie können konventionelle 
Aktienfonds ihre Treibhausgasintensität daher noch stark optimieren. Andererseits zeigt sich auch, dass einige Nachhaltigkeits- und Klimafonds ihre Portfolios noch auf ihren Carbon Footprint hin überprüfen sollten. Innerhalb der nachhaltigen und klimafreundlichen Fonds ergeben sich Streuungen der Treibhausgasintensitäten von bis zu 400 Prozent. Einzelne Fonds weisen sogar höhere Treibhausgasemissionen auf als die durchschnittlichen Standardfonds.

\section{Kapitalanlagen bieten enorme Potenziale für den Klimaschutz}

Durch die Wahl klimafreundlicher Finanzdienstleistungen können private Anleger ihren Carbon Footprint um etwa 42 Prozent senken. In den einzelnen Produktkategorien lassen sich Einsparungen zwischen 35 Prozent und 87 Prozent realisieren. Vergleichbar ist dies etwa mit dem Umstieg vom Auto auf den öffentlichen Personennahverkehr. Hier beträgt die Verringerung des durchschnittlichen Carbon Footprint zwischen 34 Prozent und 50 Prozent.

Zwar gilt es noch verschiedene methodische und statistische Hürden zu überwinden, bevor der Carbon Footprint präzise und verlässliche Zahlen liefern kann, und weitere konzeptionelle Arbeiten werden erforderlich sein, um mit dem Instrument besser operieren zu können. Aber die Ermittlung der Intensitäten der finanzierten Emissionen bietet im Grundsatz eine wertvolle Unterstützung für Privatanleger. Sie zeigt den Einfluss der Kapitalanlage auf den Klimawandel auf. Perspektivisch eignet sich dies außerdem für Finanzdienstleister zur Risikooptimierung. Durch die Integration der Berechnung von Treibhausgasintensitäten in die Kapital- und Kreditvergabe können sie die Risiken von klimapolitischen Vorgaben und Energiepreissteigerungen für ihre Investitionen besser einschätzen.

\section{Appell an Finanzdienstleister}

Die Studie „Der Carbon Footprint von Kapitalanlagen“ ist somit ein Appell an alle Marktteilnehmer, das Thema Klimaschutz und Finanzdienstleistungen weiter aktiv voranzutragen und die Chancen zu nutzen, die sich mit einer klimafreundlichen Kapitalanlage für alle verknüpfen (2).

\section{Anmerkungen}

(1) Alle Treibhausgasintensitäten werden in Kohlenstoffdioxid-Äquivalenten in Gramm pro investierten Euro im Jahr ausgewiesen.

(2) Wendler, D. / Kahlenborn, W. / Dierks, H.: Der Carbon Footprint von Kapitalanlagen. Ermittlung der Treibhausgasintensität der Kapitalanlage privater Haushalte. Berlin 2010. Download unter http://www.klima-und-finanzen.de/klimaund-finanzen/klimaschutzpotenziale-vonkapitalanlagen.html

I AUTOREN + KONTAKT

Romy Fiedler und Daniel Wendler sind Projektmanager bei adelphi, Walter Kahlenborn ist Mitbegründer und Geschäftsführer von adelphi.

Romy Fiedler, adelphi, Caspar-Theyß-Straße 14a, 14193 Berlin. E-Mail: fiedler@adelphi.de, Internet: http://www.adelphi.de

\section{politische ökologie}

Die Zeitschrift für Querdenker und Vordenkerinnen

Nanotechnologie

Aufbruch ins Ungewisse

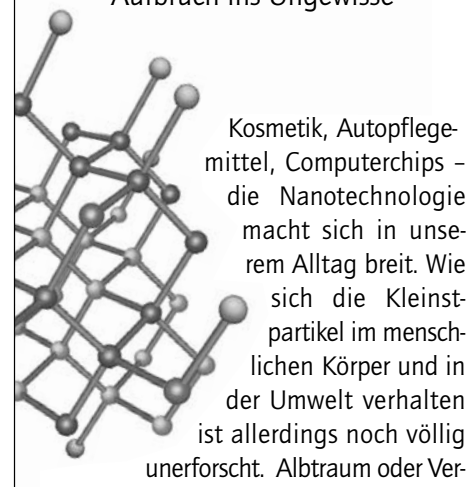

heißung - die Autorinnen und Autoren der politischen ökologie 101 klären auf über die Chancen und Risiken der neuen Basistechnologie und diskutieren Vorschläge einer politischen Regulierung.

Welche ethischen Fragen wirft die Nanotechnologie auf?

Sind Nano-Produkte öko-effizienter? _Welche Nano-Visionen sind berechtigt, welche nur Science-Fiction?

Mit Beiträgen von A. und K. Steinmüller, A. Nordmann, A. Reller, P. Mooney, U. Lahl, A. von Gleich, P. Schaper-Rinkel u.v.m.

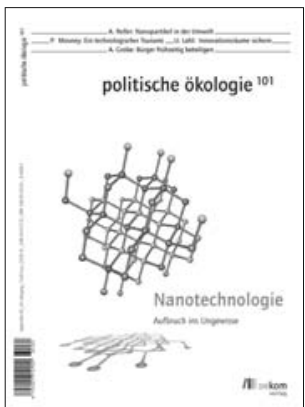

Stillen Sie Ihre Neugier!

_Fordern Sie Heft 101 an!

14,90 EUR (inkl. Versand)/23,80 CHF

_ISBN 3-86581-050-0

_oekom verlag

_Fax +49/(0)81 91/970 00-103

www.oekom.de

neugier@oekom.de 


\section{Lizenzhinweis}

Die Beiträge in ÖkologischesWirtschaften werden unter der Creative-Commons-Lizenz "CC 4.0 Attribution Non-Commercial No Derivatives" veröffentlicht. Im Rahmen dieser Lizenz muss der Autor/Urheber stets genannt werden, das Werk darf nicht bearbeitet, abgewandelt oder in anderer Weise verändert und außerdem nicht kommerziell genutzt werden. Die digitale Version des Artikels bleibt für zwei Jahre Abonnent/innen vorbehalten und ist danach im Open Access verfügbar. 